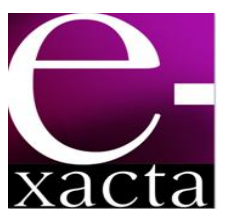

\title{
APLICAÇÃO DE CARVÃO ATIVADO IMPREGNADO COM ÍONS DE ZINCO PARA REMOÇÃO DE CISTOS DE GIARDIA SPP.
}

\author{
APPLICATION OF ACTIVATED CARBON IMPREGNATED WITH ZINC IONS \\ FOR REMOVAL OF GIARDIA SPP. CYSTS
}

\author{
Quelen Letícia Shimabuku'; Flávia Sayuri Arakawa²; Franciele Camacho ${ }^{3}$; Leticia Nishi; \\ Carole Silveira $^{5}$; Rosangela Bergamasco ${ }^{6}$
}

1 Mestre em Engenharia Química. Universidade Estadual de Maringá, 2011. Doutoranda em Engenharia Química PEQ/UEM. Maringá, PR.le.shimabuku@gmail.com

2 Mestre em Engenharia Química. Universidade Estadual de Maringá, 2011. Doutoranda em Engenharia Química PEQ/UEM. Maringá, PR. fláviasayuri@gmail.com

3 Mestre em Engenharia Química. Universidade Estadual de Maringá, 2012. Doutoranda em Engenharia Química PEQ/UEM. Maringá, PR. franciele camacho@hotmail.com

4 Doutora em Engenharia Química. Universidade Estadual de Maringá, 2011.leticianishi@hotmail.com

5 Mestranda em Engenharia Química. PEQ/UEM. Universidade Estadual de Maringá, PR .carole silveira@hotmail.com

6 Doutora em Engenharia Química. Universidad.e Estadual de Maringá, 1984. Professora da Universidade Estadual de Maringá, PR. ro.bergamasco@hotmail.com

Recebido em: 20/04/2013 - Aprovado em: 30/05/2013 - Disponibilizado em: 11/06/2013

RESUMO: Este trabalho teve como objetivo o estudo de filtros de carvão ativado impregnado com zinco para remoção do protozoário parasita Giardia. Para a impregnação dos íons metálicos na superfície do carvão ativado foi utilizado o método de impregnação por excesso de solvente, para a caracterização textural do material produzido foram analisados os parâmetros: área superficial específica BET, área de microporos (método t), volume e diâmetro de microporos (método HK). A caracterização estrutural foi feita através de medidas de Difração de Raios- $X$ (DRX). Os filtros de carvão ativado impregnado com zinco foram testados com água sintética (água deionizada contaminadas com cistos de Giardia, em uma concentração na ordem de $10^{5} \mathrm{cistos} / \mathrm{L}$ ). Os resultados desse trabalho mostraram que filtros de carvão ativado impregnados com zinco atingiram uma remoção de aproximadamente 100\%, trazendo resultados promissores na remoção do protozoário parasita Giardia.

Palavras-chave: Carvão ativado. Impregnação. Giardia. Zinco.

ABSTRACT: This work aimed to study the activated carbon filters impregnated with zinc for removal of protozoan parasite Giardia. For impregnation of the metal ions on the surface of activated charcoal was used the method of impregnation by excess of solvent, for textural characterization of the materials produced were analyzed parameters: BET specific surface area, area of micropores (method t), diameter and volume micropores (HK method). Structural characterization was made through measurements of $X$-ray diffraction (XRD). The activated carbon filters impregnated with zinc were tested with synthetic water (water contaminated with Giardia cysts, with a concentration on the order of 105 cysts / L). The results of this study showed that activated carbon filters impregnated with zinc reached a removal of approximately 100\%, bringing promising results in the removal of the protozoan parasite Giardia

KEYWORDS: Activated carbon. Impregnation. Giardia. Zinc. 


\section{INTRODUÇÃo}

Giardia e Cryptosporidium são protozoários patogênicos de importância mais expressiva na transmissão hídrica e apresentam distribuição mundial causando distúrbios gastrointestinais no homem e nos animais (THOMPSON, 2000; FAYER; MORGAN; UPTON, 2000; GARDNER; HILL, 2001; LUMADUE et al., 2001).

Quando o tratamento da água potável é inadequado podem conter números de parasitas suficientes para causar doença. A maioria dos surtos notificados associados com estes agentes protozoários patogênicos ocorrem através da água (CARVALHOALMEIDA et al., 2006;. FRANCO; CORDEIRO, 1996; GONÇALVES et al., 2006).

No Brasil, criptosporidiose e giardíase representam uma importante causa de morbidade em crianças de 0 a 5 anos (CARVALHO-ALMEIDA et al., 2006;. FRANCO; CORDEIRO, 1996; GONÇALVES et al., 2006). De acordo com Mascarini e Donalísio (2006) há alta prevalência de giardíase entre crianças que são atendidas em creches no Brasil, principalmente nas localizadas em áreas de baixa renda econômica.

Destaca-se a preocupação com a presença de oocistos de Cryptosporidium spp. e cistos de Giardia spp., devido à resistência destas estruturas ao processo de desinfecção. Ambos são passíveis de remoção por meio da filtração, mas somente à custa de um rigoroso controle desse processo (LECHEVALLIER; KWOK-KEUG, 2004).

O carvão ativado é conhecido como um material poroso de elevada área superficial específica, representando um dos grupos de adsorventes mais importantes do ponto de vista industrial. Este tipo de material possui atraentes propriedades de adsorção, que têm sido utilizadas para purificação e eliminação de componentes tóxicos em fases líquidas e gasosas, além do emprego em reações de catálise. Devido à degradação progressiva do meio ambiente, é esperado que o carvão ativado passe a desempenhar um importante papel na redução de poluentes (HAYASHI et al., 2002, ISMADJl et al., 2005).

Tem sido sugerido que os metais pesados podem bloquear os sistemas enzimáticos de microrganismos ou interferir com alguns metabólitos essenciais celulares de bactérias e protozoários (MORGAN LACKEY, 1958).

No presente estudo foi utilizado carvão ativado modificado com íons metálicos zinco no procedimento de filtração para remoção de Giardia da água e foi comparada com o carvão ativado sem a impregnação de íons metálicos, avaliando a influência dos íons metálicos na remoção do protozoário.

\section{Metodologia}

\subsection{Preparação do carvão ativado IMPREGNADO COM ZN ${ }^{2+}$}

Os filtros utilizados para avaliação da eficiência de remoção de Giardia foram preparados com carvão ativado granular (20×40 mesh) de origem vegetal (casca de coco de dendê) com e sem impregnação de íons de zinco na superfície do carvão ativado granular. Foi utilizado o métodos de impregnação por excesso de solvente e empregadas concentrações de 0,5, 1,0, 3,0 e 5,0\% em massa de zinco em relação à massa de carvão.

O carvão ativado foi colocado no balão do evaporador rotativo e foi adicionada água deionizada em proporção 1:1, sendo $200 \mathrm{~g}$ de carvão ativado em 200 $\mathrm{mL}$ de água. Em seguida, o sal $\mathrm{ZnCl}_{2}$ foi dissolvido em $30 \mathrm{~mL}$ de água deionizada e a solução foi colocada no balão. Essa mistura foi mantida sob rotação de $20 \mathrm{rpm}$ a $60^{\circ} \mathrm{C}$ por 24 horas. $\mathrm{O}$ excesso de água foi retirado a vácuo e a amostra foi seca em estufa a $100^{\circ} \mathrm{C}$ por 24 horas. O carvão seco foi levado à mufla para 
calcinação, com a finalidade de fixar os íons metálicos na sua superfície e eliminar os ânions dos sais metálicos que ocupam os sítios de adsorção, os quais deveriam estar livres (KUMAR et al., 2004).

\subsection{CARACTERIZAÇÃo do CARVÃo ATIVADO}

A caracterização textural do carvão ativado puro e das amostras impregnadas com os íons metálicos foi realizada em um Sistema de sorção de gases Quantachrome, pela adsorção/dessorção de nitrogênio $\left(\mathrm{N}_{2}\right)$ a $77 \mathrm{~K}$, seguindo a técnica básica recomendada pela IUPAC para a caracterização da estrutura porosa de adsorventes (GAUDEN et al., 2006). Deste equipamento foram obtidos dados da área superficial BET, área de microporos (método t), volume e diâmetro de microporos (método HK).

As análises de difração de raios-X (DRX) para as amostras de carvão ativado com e sem a impregnação de íons metálicos foram obtidas através de um difratômetro (Shimadzu) utilizando radiação CuKa (PARK; JANG, 2003).

\subsection{Preparo dA SUSPENSÃo de GIARDIA}

A água deionizada foi contaminada artificialmente com solução de Giardia ssp. em uma concentração que variava entre $2,0 \times 10^{5}$ e $2,5 \times 10^{5}$ cistos/L, respectivamente (EMELKO, 2004; GITS; HAUGHT; KRISHNAN, 2002).

Os ensaios para avaliação da eficiência microbiológica foram realizados através de uma adaptação da Norma Brasileira ABNT NBR 15176 - Aparelho para melhoria da qualidade da água de uso doméstico - Aparelho por gravidade (ABNT, 2004), onde as análises são realizadas no início (5\%) e final (95\%) da vida útil dos filtros, onde a vida útil considerada foi de 400 litros, conforme marca de filtros existentes no mercado consumidor.

\subsection{AvaliaÇÃo dA EFICIÊNCIA dE REMOÇÃO DE GIARDIA}

As amostras de água foram coletadas na saída do filtro e avaliadas em relação à presença de Giardia spp. pela Técnica de Filtração em Membrana com extração mecânica e eluição (CANTUSIO NETO; FRANCO, 2004). A técnica consiste em filtrar as amostras em membranas de acetato de celulose (Millipore ${ }^{\circledR}$ ) de $47 \mathrm{~mm}$ de diâmetro e 1,2 $\mu \mathrm{m}$ de porosidade. Em seguida é feita a extração mecânica do material retido na membrana com o auxílio de espátulas plásticas, alternando com lavagens da superfície da membrana durante 10 minutos em placa de Petri com solução de eluição de Tween 80 a 1\% (v/v) por 2 minutos, aspergindo com a Pipeta Pasteur, repetindo esse procedimento 2 vezes para extrair a maior quantidade possível do material retido.

A suspensão celular foi centrifugada a $600 \times \mathrm{g}$ por 15 minutos. O sobrenadante foi descartado com auxílio de Pipetas Pasteur até o volume de $3 \mathrm{~mL}$ e 0 sedimento suspenso novamente em água destilada, completando $15 \mathrm{~mL}$ do tubo de centrífuga e, a seguir, centrifugado novamente a $600 \times \mathrm{g}$ por 15 minutos. $\mathrm{O}$ sobrenadante foi descartado até obter um volume de 1 $\mathrm{mL}$ no tubo de centrífuga e o sedimento novamente suspenso nesse volume, sendo acondicionados em microtubos de $1,5 \mathrm{~mL}$.

A partir desse volume final foi realizada a leitura de Giardia spp. pela análise da solução por imunofluorescência direta (IF) com a utilização do Kit comercial Merifluor (Meridian Bioscience, Cincinnatti, Ohio).

Para estimar a recuperação e eficiência de remoção de cistos/L foi utilizada as equações 1 e 2 , respectivamente (CANTUSIO NETO; FRANCO, 2004):

$$
X=\frac{n^{\circ} \text { de cistos } \times 10^{6} \times \text { volume do pellet }(1 \mathrm{~mL})}{\text { volume utilizado/lâmina } \times \text { volume da amostra }(\mathrm{mL})}
$$




$$
Y=\quad \frac{n^{\circ} \text { de cistos } \times 100}{n^{\circ} \text { de cistos semeados }}
$$

\section{Resultados e discussão}

\subsection{Caracterização do CARVÃo ativado}

Os efeitos da impregnação de $\mathrm{Zn}^{2+}$ nas propriedades texturais do carvão ativado são mostrados na Tab.

1. Foram analisados a área específica de superfície $\left(S_{B E T}\right)$, área de microporos $\left(S_{m}\right)$, volume de poros $\left(V_{P}\right)$ e diâmetro médio de poros $\left(D_{P}\right)$, para as amostras de carbono sem, e com impregnação de íons $Z^{2+}$. A área superficial específica do carvão ativado impregnado com o íon metálico diminuiu em relação ao carvão ativado sem a impregnação, como era esperado, estes valores foram coerentes com valores encontrados para carvão ativado comercial como reportado por Freitas, Mendes e Coelho, (2007). Analisando os valores de área de superfície específica e a área de microporos observam-se que as amostras têm principalmente microporos, que de acordo com a classificação da IUPAC (União Internacional de Química Pura e Aplicada), possuem diâmetro de poros inferior a $20 \AA$ (GREGG; SING, 1982). O volume de poros nas amostras impregnadas diminuiu sugerindo que os íons metálicos foram depositados nos poros.

Tabela 1

Propriedades texturais do carvão com e sem a impregnação de íons metálicos $\mathrm{Zn}^{2+}$

\begin{tabular}{ccccc}
\hline Amostra & $\mathrm{S}_{\mathrm{BET}}\left(\mathrm{m}^{2} \mathrm{~g}^{-1}\right)$ & $\mathrm{S}_{\mathrm{m}}\left(\mathrm{m}^{2} \mathrm{~g}^{-1}\right)$ & $\mathrm{V}_{\mathrm{p}}\left(\mathrm{cm}^{3} \mathrm{~g}^{-1}\right)$ & $\mathrm{dp}(\AA)$ \\
\hline C puro & 870,00 & 743,00 & 0,4227 & 16 \\
$\mathrm{C} / \mathrm{Zn} 0,5 \%$ & 823,00 & 737,00 & 0,3266 & 15 \\
$\mathrm{C} / \mathrm{Zn} 1,0 \%$ & 723,00 & 671,00 & 0,3433 & 15 \\
$\mathrm{C} / \mathrm{Zn} 3,0 \%$ & 691,00 & 596,00 & 0,3405 & 14 \\
$\mathrm{C} / \mathrm{Zn} 5,0 \%$ & 667,00 & 579,00 & 0,3601 & 14 \\
\hline
\end{tabular}

Os difratrogramas de raios-X são mostrados na Fig. 1. resultados das medidas de difração de raios-X Os picos foram localizados nos valores de $2 \theta$ variando fornecida pelo software JCPDS. entre 20 a $70^{\circ}$. Na Tab. 2, seguem as atribuições dos

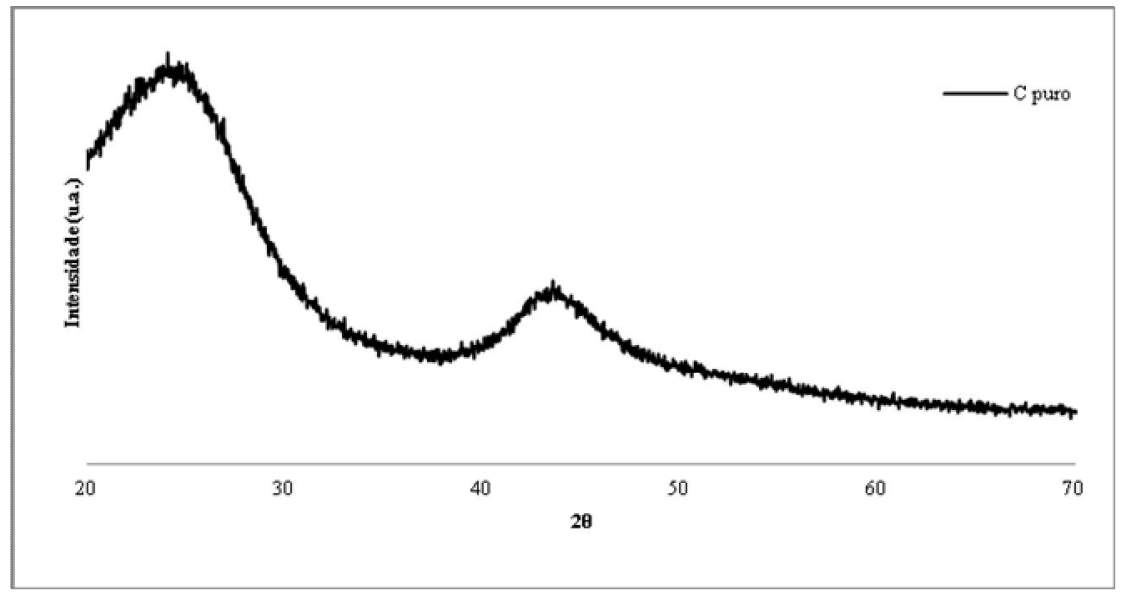

(a) 


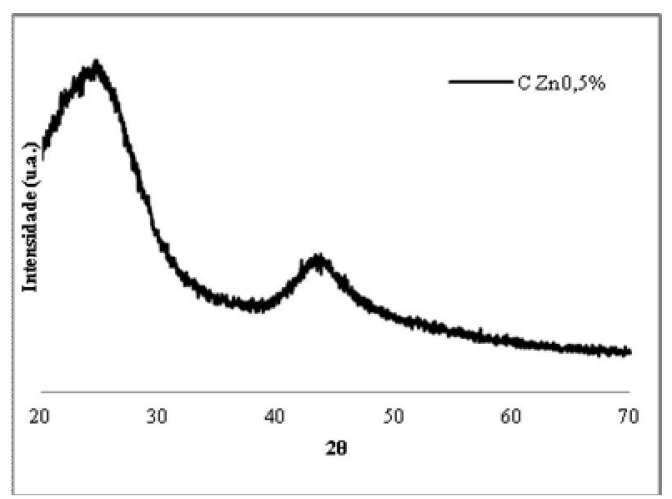

(b)

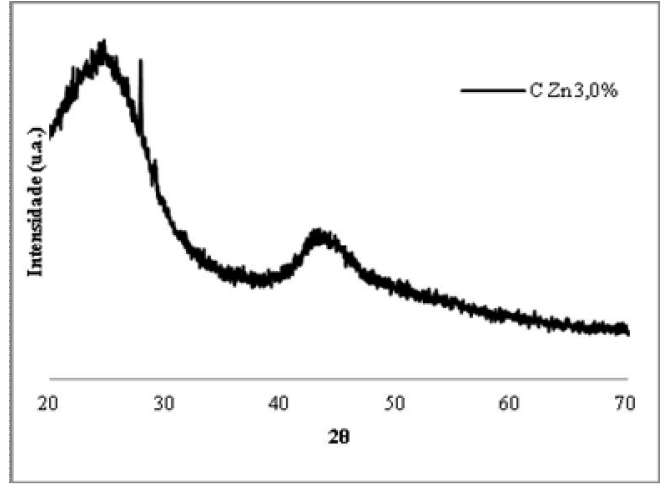

(d)

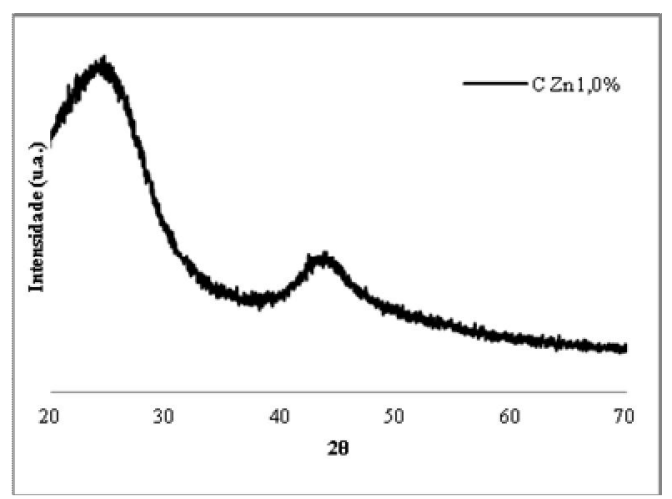

(c)

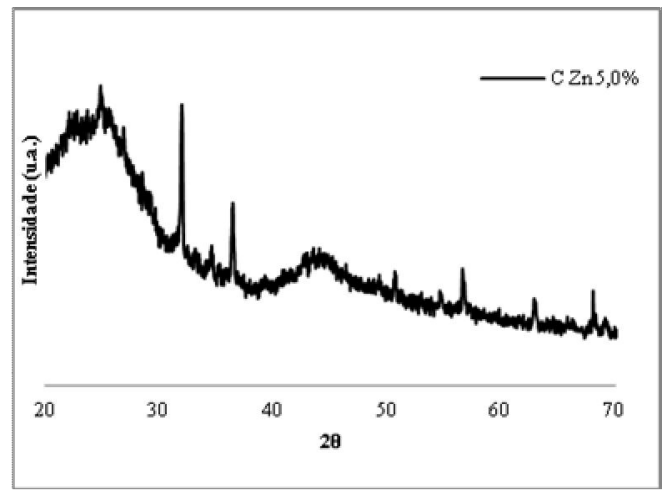

(e)

Figura 1 - Difratogramas de raios-X para amostras de carvão ativado puro (a) e impregnadas com 0,5\% (b), 1,0\% (c), $3,0 \%$ (d) e $5,0 \%$ (e) de $\mathrm{Zn}^{2+}$

Tabela 2

Medidas de difração de raios-X para as amostras impregnadas com íons $\mathrm{Zn}^{2+}$

\begin{tabular}{ccccc}
\hline Amostra & $2 \theta$ & $\mathrm{d}(\AA)$ & $\mathrm{I}$ & Atribuição \\
\hline C puro & 27,43 & 3,249 & 6 & Carbono \\
& 44,39 & 2,039 & 6 & Carbono grafite \\
$\mathrm{C} / \mathrm{Zn} 0,5 \%$ & 23,98 & 3,710 & 40 & Carbono \\
& 44,39 & 2,039 & 6 & Carbono grafite \\
$\mathrm{C} / \mathrm{Zn} \mathrm{1,0 \%}$ & 23,98 & 3,710 & 40 & Carbono \\
& 44,39 & 2,039 & 6 & Carbono grafite \\
$\mathrm{C} / \mathrm{Zn} \mathrm{3,0 \%}$ & 23,98 & 3,710 & 40 & Carbono \\
& 26,26 & 3,390 & 10 & Oxido de zinco \\
& 44,39 & 2,039 & 6 & Carbono grafite \\
$\mathrm{C} / \mathrm{Zn} \mathrm{5,0 \%}$ & 23,98 & 3,710 & 40 & Carbono \\
& 26,62 & 3,348 & 100 & Carbono \\
& 27,43 & 3,249 & 6 & Carbono
\end{tabular}




\begin{tabular}{cccc}
31,77 & 2,814 & 57 & Óxido de zinco \\
34,42 & 2,603 & 44 & Óxido de zinco \\
36,25 & 2,475 & 100 & Óxido de zinco \\
44,39 & 2,039 & 6 & Carbono grafite \\
50,72 & 1,800 & 5 & Carbono grafite \\
54,62 & 1,680 & 50 & Óxido de zinco \\
56,60 & 1,625 & 32 & Óxido de zinco \\
62,86 & 1,477 & 29 & Óxido de zinco \\
66,38 & 1,407 & 4 & Óxido de zinco \\
67,96 & 1,378 & 23 & Óxido de zinco \\
69,10 & 1,358 & 11 & Óxido de zinco \\
\hline
\end{tabular}

As amostras de carvão ativado puro, 0,5 e 1,0\% de zinco apresentaram em sua estrutura os mesmos planos basais em valores $2 \theta$ próximos a $27^{\circ}$ correspondente ao carbono e em $44^{\circ}$ correspondente ao carbono grafite. Nestas amostras nenhuma interferência de zinco foi encontrada, a impregnação dos íons metálicos pode não ter ocorrido de forma homogênea e, assim, a amostra utilizada para análise não apresentou traços de zinco. Para a amostra de $3,0 \%$ de zinco, foi identificado um pico de óxido de zinco em $26,26^{\circ}$. Para a amostra impregnada com $5,0 \%$ de zinco, foram encontrados picos entre $31,77^{\circ} \mathrm{e}$ $69,10^{\circ}$ correspondentes a óxido de zinco.

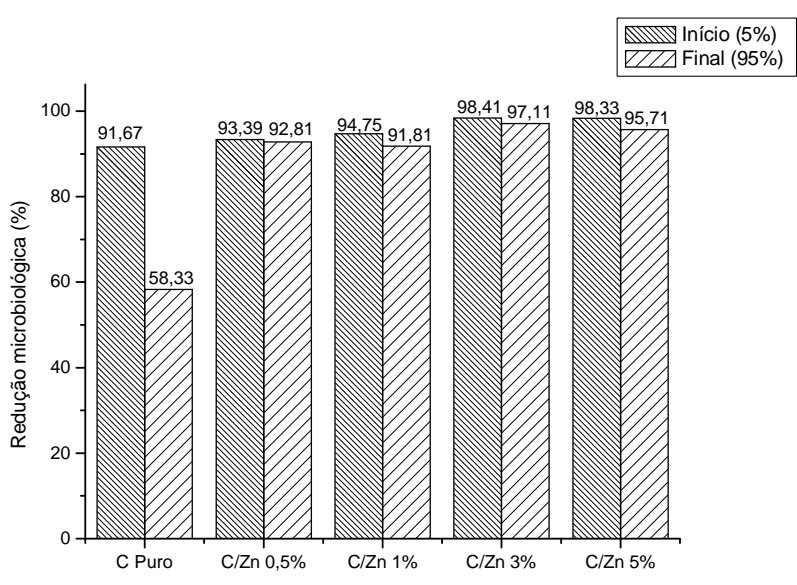

Figura 2 - Eficiência de remoção para amostras de carvão ativado puro e impregnadas com $0,5 \%, 1,0 \%$, $3,0 \%$ e $5,0 \%$ de $\mathrm{Zn}^{2+}$

\subsection{EFICIÊNCIA DE REMOÇÃO DE GIARDIA}

A Fig. 2 mostra a porcentagem de remoção de Giardia spp. para as amostras em diferentes concentrações de $\mathrm{Zn}^{2+}$.

Para os ensaios com carvão ativado granular, sem a presença de $\mathrm{Zn}^{2+}$ foi observada uma redução de $91,67 \%$ e $58,33 \%$ no início e no final do experimento, respectivamente. Para as amostras de carvão ativado impregnado com íons $\mathrm{Zn}^{2+}$ em uma concentração de 0,5, 1,0, 3,0 e 5,0\% em massa, foram obtidas reduções de 93,39 e $92,81 \%, 94,75$ e $91,81 \%$, 98,41 e $97,71 \%, 98,33$ e $95,71 \%$ no início (5\%) e final (95\%) da vida útil do filtro, respectivamente.

Através dos resultados obtidos observou-se que a presença do metal no carvão ativado influenciou a eficiência de remoção dos filtros, uma vez que utilizando o carvão ativado sem a presença dos íons metálicos houve uma grande redução na eficiência de remoção no final de sua vida útil. Ao contrário, as amostras com a presença dos íons $\mathrm{Zn}^{2+}$ se mostraram eficientes na remoção do protozoário, tanto no início (5\%) como no final (95\%) da vida útil do filtro.

Estudos com carvão ativado impregnado com íons metálicos para remoção de protozoários da água estão em desenvolvimento, não sendo encontrados resultados para comparação de eficiência de remoção. 
As eficiências de remoção obtidas foram superiores a $91 \%$, resultados semelhantes foram reportado por Cheng et al. (2009); Medena e Schijven (2001) em seus estudos para remoção de protozoários, com diferentes processos de tratamento.

\section{Conclusões}

A caracterização dos meios porosos produzidos mostrou que a impregnação de íons metálicos em sua superfície causou um comportamento geral para todas as amostras, os parâmetros estudados diminuíram de acordo com o aumento das concentrações utilizadas, quando comparadas a amostras de carvão ativado sem o íon metálico impregnado.

Através dos resultados obtidos nesta pesquisa, podese afirmar que a presença de metais na superfície do carvão ativado influenciou a remoção de Giardia da água, apresentando resultados significativos quando comparadas a sistemas de tratamento utilizados para remoção dos mesmos. Assim, podem-se utilizar esses filtros como um tratamento alternativo ou complementar ao tratamento convencional na remoção de protozoários para obtenção de água livre destes contaminantes.

\section{REFERÊNCIAS}

ABNT - Associação Brasileira de Normas Técnicas. NBR 15176: Aparelho para melhoria da qualidade da água de uso doméstico - Aparelho por gravidade, 2004.

BARWICK, R. S., LEVY, D. A., CRAUN, G. F., BEACH, M. J., CALDERON, R. L. Surveillance for waterborne-disease outbreaks-United States, 19971998. MMWR 49, 1-21, 2000.

CANTUSIO NETO, R.; FRANCO, R. M. B. Ocorrência de oocistos de Cryptosporidium spp. e cistos de Giardia spp. em diferentes pontos do processo de tratamento de água, em Campinas, Säo Paulo, Brasil. Rev Hig. Aliment., n. 118, p. 52-59, 2004.

CARVALHO-ALMEIDA, T. T, PINTO, P. L. S., QUADROS, C. M. S., TORRES, M. A. G. V., KANAMURA, H. Y., CASIMIRO, A. M. Detection of Cryptosporidium sp. in non-diarrheal faeces from children in a day care center in the city of São Paulo, Brazil. Rev Inst Med Trop Sao Paulo; 48(1):27-32, 2006.

CHENG, H. W.; LUCY, F. E.; GRACZYK, T. K.; BROADERS, M. A.; TAMANG, L.; CONNOLLY, M. Fate of Cryptosporidium parvum and Cryptosporidium hominis oocysts and Giardia duodenalis cysts during secondary wastewater treatments. Parasitology Reseach. v. 105, p. 689-696, 2009.

CRAUN, G. F., HUBBS, S. A., FROST, F., CALDERON, R. L., VIA, S. H. Waterborne outbreaks of cryptosporidiosis. J. Am. Water Works. Assoc. 90, 81-91, 1998.
EMELKO, M. B. Removal of viable and inactivated Cryptosporidium by dual and tri media filtration. Water Research. v. 37, p. 2998-3008, 2004.

FAYER, R., MORGAN, U., UPTON, S. J. Epidemiology of Cryptosporidium: transmission, detection and identification. International Journal for Parasitology, n.30, p. 1305-1322, 2000.

FRANCO, R. M. B., CORDEIRO, N. S. S. Giardiasis and cryptosporidiosis in day-care centers in the municipality Campinas SP. Rev Soc Bras Med Trop; 29(6):585-91, 1996.

FREITAS, A. F.; MENDES, M. F.; COELHO, G. L. V. Thermodynamic study of fatty acids adsorption on different adsorbents. Journal of Chemical Thermodynamics, v. 39, p. 1027-1037, 2007.

GARDNER, T. B., HILL, D. R. Treatment of giardiasis. Clin. Microbiol. Rev. 14, 114-128, 2001.

GAUDEN, P. A., SZMECHTIG-GAUDEN, E., RYCHLICKI, G., DUBER, S., GARBACZ, J. K., BUCZKOWSKI, R. Changes of the porous estructure of activated carbons applied in a filter bed pilot operation. J. Colloid Interface Sci., v. 295, p. 327-347, 2006.

GITS, V., HAUGHT, R. C., KRISHNAN, E. R. Depressed filtration ripening enhances removal of Cryptosporidium parvum. Water Science and Technology: Water Supply. v. 2, n. 3, p. 159-168, 2002. 
GONÇALVES, E. M. N., SILVA, A. J., EDUARDO, M. B. P., UEMURA, I. H., MOURA, I. N. S., CASTILHO, V. L. P., CORBETT, C. E. P. Multilocus genotyping of Cryptosporidium hominis associated with diarrhea outbreak in a day care unit in São Paulo. Clinics; 61(2):119-26, 2006.

GREGG, S. J., SING, K. S. W. Adsorption, Surface Area and Porosity. $2^{\mathrm{a}}$ Ed. London - England. Academic Press. p. 40-48, 1982.

HAYASHI, J., HORIKAWA, T., MUROYAMA, K., GOMES, V. G. Activated carbon from chickpea husk by chemical activation with $\mathrm{K}_{2} \mathrm{CO}_{3}$ : preparation and characterization. Microporous and Mesoporous Materials. v. 55, p. 63-68, 2002.

ISMADJI, S., SUDARYANTO, Y., HARTONO, S. B., SETIAWAN, L. E. K., AYUCITRA, A. Activated carbon from char obtained from vacuum pyrolysis of teak sawdust: pore structure development and characterization. Bioresource Technology. v. 96, p. 1364-1369, 2005.

KUMAR, V. S., NAGARAJA, B. M., SHASHIKALA, V., PADMASRI, A. H., MADHAVENDRA, S. S., RAJU, B. D., RAO, K.S.R. Highly efficient Ag/C catalyst prepared by electro-chemical deposition method in controlling microorganisms in water. J. Mol. Catal. A: Chem., v. 223, pp. 313-319, 2004.

LeCHEVALLIER, M. W., KWOK-KEUNG, A. U. Water treatment and pathogen control Process: Efficiency in achieving safe drinking water. London: WHO/IWA, 2004.
LEE, S. H., LEVY, D. A., CRAUN, G. F., BEACH, M. J., CALDERON, R. L. Surveillance for waterbornedisease outbreaks-United States, 1999-2000. MMWR 51, 1-47, 2002.

LUMADUE, J. A., MANABE, Y. C., MOORE, R. D., BELITSOS, P. C., SEARS, C. L., CLARK, D. P. A clinicopathologic analysis of AIDS-related cryptosporidiosis. AIDS 12, 2459-2466, 2001.

MASCARINI, L.M., DONALISIO, M. R. Giadiasis and cyptosporidosis in children institutionalized at daycare centers in the state of São Paulo. Rev Soc Bras Med Trop; 39(6): 577-9, 2006.

MEDENA, G. J., SCHIJVEN, J. F. Modelling the Sewage Discharge and Dispersion of Cryptosporidium and Giardia in Surface Water. Water Research. v. 35, n. 18, p. 4307-4316, 2001.

MORGAN, G. B., LACKEY, J. B. BOD determination in wastes containing chelated copper and chromium. Sewage Ind. Wastes. v. 30, p. 283-286, 1958.

PARK, S.-J., JANG, Y.-S. Preparation and characterization of activated carbon fibers supported with silver metal for antibacterial behavior. J. Colloid Interface Sci., v. 261, p. 238-243, 2003.

THOMPSON, R. C. A. Giardiasis as re-emerging infectious disease and its zoonotic potential. International Journal for Parasitology. v. 30, p. 12591267, 2000. 